\title{
Degree 4 unramified cohomology with finite coefficients and torsion codimension 3 cycles
}

\author{
Claire Voisin
}

CNRS, Institut de mathématiques de Jussieu

\section{Introduction}

Let $X$ be a smooth complex projective variety and $A$ an abelian group. Degree $i$ unramified cohomology $H_{n r}^{i}(X, A)$ of $X$ with coefficients in $A$ can be defined as the direct limit of the sets of data

$$
\alpha_{k} \in H_{B}^{i}\left(U_{k}, A\right), \alpha_{k \mid U_{k} \cap U_{l}}=\alpha_{l \mid U_{k} \cap U_{l}},
$$

where the $U_{k}$ 's are sufficiently small Zariski open sets covering $X$. Here the notation $H_{B}^{i}$ stands for Betti cohomology of the underlying complex analytic space. In other words,

$$
H_{n r}^{i}(X, A)=H^{0}\left(X_{Z a r}, \mathcal{H}^{i}(A)\right)
$$

where the sheaf $\mathcal{H}^{i}(A)$ on $X_{Z a r}$ is associated to the presheaf $U \mapsto H_{B}^{i}(U, A)$.

We refer to section 1 for a more detailed review of Bloch-Ogus theory, which naturally leads to the introduction of the sheaves $\mathcal{H}^{i}(A)$. The important feature of unramified cohomology is the fact that it provides us with birational invariants of $X$, which vanish for projective space: in fact, these groups are even stable birational invariants (cf. [7]), that is, they are invariant under the relation :

$$
X \cong Y \text { if } X \times \mathbb{P}^{r} \text { is birationally equivalent to } Y \times \mathbb{P}^{s} \text { for some } r, s \text {. }
$$

Unramified cohomology with torsion coefficients (that is, $A=\mathbb{Z} / n \mathbb{Z}$ or $A=\mathbb{Q} / \mathbb{Z}$ ) plays an important rôle in the study of the Lüroth problem, that is the study of unirational varieties which are not rational, (sse for example the papers [1], [7], [17]). In fact, the invariant used by Artin-Mumford, which is the torsion in the group $H_{B}^{3}(X, \mathbb{Z})$, is equal for rationally connected varieties to the unramified cohomology group $H_{n r}^{2}(X, \mathbb{Q} / \mathbb{Z})$. In the paper [7], the authors exhibit unirational sixfolds with vanishing group $H_{n r}^{2}(X, \mathbb{Q} / \mathbb{Z})$ but non vanishing group $H_{n r}^{3}(X, \mathbb{Q} / \mathbb{Z})$. Their example is reinterpreted in the recent paper [8], using the following groups $Z^{2 i}(X)$ which are proved in [20] to be birational invariants of $X$ for $i=2$ and $i=\operatorname{dim} X-1$ :

$$
Z^{2 i}(X):=\frac{H d g^{2 i}(X, \mathbb{Z})}{H_{B}^{2 i}(X, \mathbb{Z})_{a l g}}
$$

where $H d g^{2 i}(X, \mathbb{Z})$ is the set of integral Hodge classes of degree $2 i$ on $X$ and $H_{B}^{2 i}(X, \mathbb{Z})_{a l g} \subset$ $H_{B}^{2 i}(X, \mathbb{Z})$ is the subgroup generated over $\mathbb{Z}$ by cycle classes.

In the paper [8], we give the following comparison result between $Z^{4}(X)$ and $H_{n r}^{3}(X, \mathbb{Q} / \mathbb{Z})$ : 
Theorem 0.1. (Colliot-Thélène and Voisin 2010) For any smooth projective $X$, there is an exact sequence

$$
0 \rightarrow H_{n r}^{3}(X, \mathbb{Z}) \otimes \mathbb{Q} / \mathbb{Z} \rightarrow H_{n r}^{3}(X, \mathbb{Q} / \mathbb{Z}) \rightarrow \operatorname{Tors}\left(Z^{4}(X)\right) \rightarrow 0 .
$$

If $C H_{0}(X)$ is supported on a closed algebraic subset of dimension $\leq 3$, Tors $\left(Z^{4}(X)\right)=Z^{4}(X)$. If $C H_{0}(X)$ is supported on a closed algebraic subset of dimension $\leq 2, H_{n r}^{3}(X, \mathbb{Z})=0$.

In particular, if $X$ is rationally connected (so that $C H_{0}(X)=\mathbb{Z}$ ), we have

$$
H_{n r}^{3}(X, \mathbb{Q} / \mathbb{Z}) \cong Z^{4}(X) .
$$

We provide in this note a similar interpretation of the group $H_{n r}^{4}(X, \mathbb{Q} / \mathbb{Z})$. The geometric group which will play the role of the group $Z^{4}(X)$ is the group $\mathcal{T}^{3}(X)$ consisting of the image in $\operatorname{Griff}^{3}(X) \subset C H^{3}(X) / a l g$ of the kernel of the Deligne cycle map restricted to the torsion subgroup of $C H^{3}(X)$ :

$$
\mathcal{T}^{3}(X):=\left[\operatorname{Ker}\left(c l_{D}: \operatorname{Tors}\left(C H^{3}(X)\right) \rightarrow H_{D}^{6}\left(X, \mathbb{Z}_{D}(3)\right)\right)\right] / \text { alg } .
$$

We will show (cf. Lemma 2.2) that $\mathcal{T}^{3}(X)$ is a birationally invariant group.

Theorem 0.2. Assume that the group $H_{B}^{5}(X, \mathbb{Z}) / N^{2} H_{B}^{5}(X, \mathbb{Z})$ has no torsion. Then there is an exact sequence

$$
0 \rightarrow H_{n r}^{4}(X, \mathbb{Z}) \otimes \mathbb{Q} / \mathbb{Z} \rightarrow H_{n r}^{4}(X, \mathbb{Q} / \mathbb{Z}) \rightarrow \mathcal{T}^{3}(X) \rightarrow 0 .
$$

Here $N^{i} H_{B}^{j}(X, A)$ is defined as the subgroup of $H_{B}^{j}(X, A)$ consisting of cohomology classes on $X$ vanishing away from a Zariski closed subset $Y \subset X$ of codimension $\geq i$.

This theorem can as above be completed as follows:

Corollary 0.3. If $\mathrm{CH}_{0}(X)$ is supported on a closed algebraic subset of dimension $\leq 3$ and $H_{B}^{5}(X, \mathbb{Z}) / N^{2} H_{B}^{5}(X, \mathbb{Z})$ has no torsion, there is an isomorphism

$$
H_{n r}^{4}(X, \mathbb{Q} / \mathbb{Z}) \rightarrow \mathcal{T}^{3}(X)
$$

Proof. Indeed, under the first assumption, it is proved in [8, Prop. 3.3] that $H_{n r}^{4}(X, \mathbb{Z})$ is identically 0 . Using the second assumption and Theorem 0.2 , we get the desired isomorphism.

The paper is organized as follows. In section 1 we recall a few facts from Bloch-Ogus theory. Section 2 gives the needed definitions concerning the notion of coniveau and states more precisely the main result. The proof of Theorem 0.2 is given in section 3. Section 4 is devoted to the description of a few non trivial examples and the construction of new ones.

Thanks. I thank the organizers of the conference "Geometry and Arithmetic" on the island of Schiermonnikoog for the invitation to speak there and to submit a paper in the proceedings. It is a pleasure to dedicate this paper to Gerard Van der Geer on the occasion of his sixtieth birthday. 


\section{Bloch-Ogus theory and unramified cohomology}

Let $X$ be a smooth projective complex variety. We will denote $X_{c l}$ the set $X(\mathbb{C})$ endowed with its classical (or Euclidean) topology, and $X_{Z a r}$ the set $X(\mathbb{C})$ endowed with its Zariski topology.

Let

$$
\pi: X_{c l} \rightarrow X_{Z a r}
$$

be the identity of $X(\mathbb{C})$. This is obviously a continuous map, and Bloch-Ogus theory [4] is the study of the Leray spectral sequence associated to this map and any constant sheaf with stalk $A$ on $X_{c l}$. $A$ will be in applications one of the following groups: $\mathbb{Z}, \mathbb{Q}, \mathbb{Q} / \mathbb{Z}$.

We are thus led to introduce the sheaves on $X_{Z a r}$

$$
\mathcal{H}^{i}(A):=R^{i} \pi_{*} A
$$

The Leray spectral sequence for $\pi$ and $A$ has terms

$$
E_{2}^{p, q}=H^{p}\left(X_{Z a r}, \mathcal{H}^{q}(A)\right) .
$$

Unramified cohomology of $X$ with value in $A$ is defined by the formula (cf. [7])

$$
H_{n r}^{i}(X, A)=H^{0}\left(X_{Z a r}, \mathcal{H}_{X}^{i}(A)\right) .
$$

The main result of the paper by Bloch and Ogus [4] is the following Gersten-Quillen resolution for the sheaves $\mathcal{H}_{X}^{i}(A)$. For any closed subvariety $D \subset X$, let $i_{D}: D \rightarrow X$ be the inclusion map and $H^{i}(\mathbb{C}(D), A)$ the constant sheaf on $D$ with stalk

$$
\lim _{\substack{\overrightarrow{U \subset D} \\ U \subset D}} H^{i}(U(\mathbb{C}), A)
$$

at any point de $D$. When $D^{\prime} \subset D$ has codimension 1, there is a map induced by the topological residue (on the normalization of $D$ ) (cf. [23, II, 6.1.1]) :

$$
\operatorname{Res}_{D, D^{\prime}}: H^{i}(\mathbb{C}(D), A) \rightarrow H^{i-1}\left(\mathbb{C}\left(D^{\prime}\right), A\right) .
$$

For $r \geq 0$, let $X^{(r)}$ be the set of irreducible closed algebraic subsets of codimension $r$ in $X$.

Theorem 1.1. ([4], Theorem 4.2) For any $A$, and any integer $i \geq 1$, there is an exact sequence of sheaves on $X_{Z a r}$

$$
0 \rightarrow \mathcal{H}_{X}^{i}(A) \rightarrow i_{X *} H^{i}(\mathbb{C}(X), A) \stackrel{\partial}{\rightarrow} \bigoplus_{D \in X^{(1)}} i_{D *} H^{i-1}(\mathbb{C}(D), A) \stackrel{\partial}{\rightarrow} \ldots \stackrel{\partial}{\rightarrow} \bigoplus_{D \in X^{(i)}} i_{D *} A_{D} \rightarrow 0 .
$$

Here the components of the maps $\partial$ are induced by the maps $\operatorname{Res}_{D, D^{\prime}}$ when $D^{\prime} \subset D$ (and are 0 otherwise). The sheaf $A_{D}$ on $D_{Z a r}$ identifies of course to the constant sheaf with stalk $H^{0}(\mathbb{C}(D), A)$.

Let us state a few consequences proved in [4]: First of all, denoting by $C H^{k}(X) /$ alg the group of codimension $k$ cycles of $X$ modulo algebraic equivalence, we get the Bloch-Ogus formula:

Corollary 1.2. ([4], Corollary 7.4) If $X$ is a smooth complex projective variety, there is a canonical isomorphism

$$
C H^{k}(X) / a l g=H^{k}\left(X_{Z a r}, \mathcal{H}^{k}(\mathbb{Z})\right) .
$$


Proof. Indeed, the Bloch-Ogus resolution is acyclic. It thus allows to compute $H^{k}\left(X_{Z a r}, \mathcal{H}^{k}(\mathbb{Z})\right)$ by taking global sections in the above resolution, which gives

$$
H^{k}\left(X_{Z a r}, \mathcal{H}^{k}(\mathbb{Z})\right)=\operatorname{Coker}\left[\partial: \oplus_{D \in X^{(k-1)}} H^{1}(\mathbb{C}(W), \mathbb{Z}) \longrightarrow \oplus_{D \in X^{(k)}} \mathbb{Z}\right]
$$

The group $\oplus_{D \in X^{(k)}} \mathbb{Z}$ is the group of codimension $k$ cycles on $X$, and to conclude, one has to check that the image of the map $\partial$ above is the group of cycles algebraically equivalent to 0 . This follows from the fact that on a smooth projective variety $\widetilde{W}$, a divisor $D$ is cohomologous to zero (hence algebraically equivalent to zero) if and only if there exists a degree 1 cohomology class $\alpha \in H^{1}(\widetilde{W} \backslash \operatorname{Supp} D, \mathbb{Z})$ such that $\operatorname{Res} \alpha=D$.

By theorem 1.1, the sheaf $\mathcal{H}^{i}(A)$ has an acyclic resolution of length $\leq i$. We thus get the following vanishing result:

Corollary 1.3. For $X$ smooth, $A$ an abelian group and $r>i$, one has

$$
H^{r}\left(X_{Z a r}, \mathcal{H}_{X}^{i}(A)\right)=0 .
$$

Concerning the structure of the sheaves $\mathcal{H}^{i}(\mathbb{Z})$, we have the following result, which is a consequence of the Bloch-Kato conjecture recently proved by Rost and Voevodsky (we refer to [5], [8], [2] for more explanations concerning the way the very important result below is deduced from the Bloch-Kato conjecture).

Theorem 1.4. The sheaves $\mathcal{H}^{i}(\mathbb{Z})$ of $\mathbb{Z}$-modules over $X_{Z a r}$ have no torsion.

The following Corollary gives an equivalent formulation of this theorem, by considering the long exact sequence associated to the short exact sequence of sheaves on $X_{c l}$

$$
0 \rightarrow \mathbb{Z} \rightarrow \mathbb{Q} \rightarrow \mathbb{Q} / \mathbb{Z} \rightarrow 0
$$

on $X_{c l}$ and the associated long exact sequence of sheaves on $X_{Z a r}$

$$
\ldots \rightarrow \mathcal{H}^{i}(\mathbb{Q}) \rightarrow \mathcal{H}^{i}(\mathbb{Q} / \mathbb{Z}) \rightarrow \mathcal{H}^{i+1}(\mathbb{Z}) \rightarrow \mathcal{H}^{i+1}(\mathbb{Q}) \rightarrow \ldots
$$

Corollary 1.5. For any integer $i$, there is a short exact sequence of sheaves on $Z_{Z a r}$

$$
0 \rightarrow \mathcal{H}^{i}(\mathbb{Z}) \rightarrow \mathcal{H}^{i}(\mathbb{Q}) \rightarrow \mathcal{H}^{i}(\mathbb{Q} / \mathbb{Z}) \rightarrow 0
$$

\section{Statement of the result}

Let $X$ be a smooth complex projective variety and $k, l$ be integers. We can consider the subgroup $N^{l} H_{B}^{k}(X, \mathbb{Z}) \subset H_{B}^{k}(X, \mathbb{Z})$ of " coniveau $l$ cohomology", defined as

$$
N^{l} H_{B}^{k}(X, \mathbb{Z})=\operatorname{Ker}\left(H_{B}^{k}(X, \mathbb{Z}) \rightarrow \lim _{\operatorname{codim} W=l} H_{B}^{k}(X \backslash W, \mathbb{Z})\right),
$$

where the $W \subset X$ considered here are the closed algebraic subsets of $X$ of codimension $l$. Introducing a resolution of singularities $\widetilde{W}$ of $W$ and its natural morphism $\tau_{W}: \widetilde{W} \rightarrow X$ to $X$, we have

$$
N^{l} H_{B}^{k}(X, \mathbb{Q})=\sum_{\operatorname{codim} W=l} \operatorname{Im}\left(\tau_{W *}: H_{B}^{k-2 l}(\widetilde{W}, \mathbb{Q}) \rightarrow H^{k}(X, \mathbb{Q})\right),
$$

as a consequence of the fact that morphisms of mixed rational Hodge structures are strict for the weight filtration, (cf. [9], [13], [23, II, Thm. 4.20]). 
From now on, we restrict to the case $k=2 l+1$. For any $W \subset X, \tau_{W}: \widetilde{W} \rightarrow X$ as above, the Gysin morphism $\tau_{W *}: H_{B}^{1}(\widetilde{W}, \mathbb{Z}) \rightarrow H_{B}^{2 l+1}(X, \mathbb{Z})$ is a morphism of Hodge structures (of bidegree $(l, l)$ ), which induces a morphism between the intermediate Jacobians

$$
\tau_{W *}: \operatorname{Pic}^{0}(\widetilde{W})=J^{1}(\widetilde{W}) \rightarrow J^{2 l+1}(X):=\frac{H_{B}^{2 l+1}(X, \mathbb{C})}{F^{l} H_{B}^{2 l+1}(X, \mathbb{C}) \oplus H_{B}^{2 l+1}(X, \mathbb{Z}) / \text { torsion }} .
$$

This map $\tau_{W *}$ is compatible in an obvious way with the Abel-Jacobi maps $\phi_{W}$ and $\phi_{X}$, defined respectively on codimension 1 and codimension $l+1$ cycles of $\widetilde{W}$ and $X$ which are homologous to 0 .

The Deligne cycle class map

$$
c l_{\mathcal{D}}^{l+1}: C H^{l+1}(X) \rightarrow H_{\mathcal{D}}^{2 l+2}(X, \mathbb{Z}(l+1))
$$

restricts to the Abel-Jacobi map $\phi_{X}^{l}$ on the subgroup of cycles homologous to 0 (cf. [23, I,12.3.3]), and in particular on the subgroup of cycles algebraically equivalent to 0 .

If $Z \in C H^{l}(X)$ is algebraically equivalent to 0 , there exist subvarieties $W_{i} \subset X$ of codimension $l$ and cycles $Z_{i} \subset \widetilde{W}_{i}$ homologous to 0 such that $Z=\sum_{i} \tau_{W_{i} *} Z_{i}$ in $C H^{l+1}(X)$. It follows from the previous considerations that $c l_{\mathcal{D}}^{l+1}$ induces a morphism

$$
c l_{\mathcal{D}, t r}^{l+1}: C H^{l}(X) / a l g \rightarrow H_{\mathcal{D}}^{2 l+2}(X, \mathbb{Z}(l+1))_{t r}:=\frac{H_{\mathcal{D}}^{2 l+2}(X, \mathbb{Z}(l+1))}{\left\langle\tau_{W *} J^{1}(\widetilde{W}), \operatorname{codim} W=l\right\rangle} .
$$

Let $\mathcal{T}^{l+1}(X):=\operatorname{Tors}\left(\operatorname{Ker} c l_{\mathcal{D}, t r}^{l+1}\right)$.

Lemma 2.1. This group identifies to the image of the subgroup Tors $\left(\operatorname{Ker} c l_{\mathcal{D}}^{l+1}\right)$ in $C H^{l+1}(X) /$ alg.

Proof. This follows from the fact that the groups of cycles algebraically equivalent to 0 modulo rational equivalence are divisible. This implies that the natural map $\operatorname{Tors}\left(C H^{i}(X)\right) \rightarrow$ $\operatorname{Tors}\left(C H^{i}(X) / a l g\right)$ is surjective for any $i$. We then use the fact that for the $\widetilde{W}$ 's introduced above, the map

$$
C H^{1}(\widetilde{W})_{a l g} \cong \operatorname{Pic}^{0}(\widetilde{W}) \rightarrow J^{1}(\widetilde{W})
$$

is an isomorphism, where $C H^{1}(\widetilde{W})_{\text {alg }} \subset C H^{1}(\widetilde{W})$ is the subgroup of cycles algebraically equivalent to 0 .

We have the following lemma :

Lemma 2.2. The group $\mathcal{T}^{3}(X)$ is a birational invariant of $X$.

Proof. It suffices to check invariance under blow-up. The Manin formulas (cf. [14], [23, II,9.3.3]) for groups of cycles modulo rational or algebraic equivalence and for Deligne cohomology of a blow-up imply that it suffices to prove that the groups $\mathcal{T}^{i}(Y)$ are trivial for $i \leq 2$ and $Y$ smooth projective. However this is an immediate consequence of the definition, of Lemma 2.1 , and of the fact that the Deligne cycle class map $c l_{\mathcal{D}}: C H^{i}(X) \rightarrow H_{\mathcal{D}}^{2 i}(X, \mathbb{Z}(i))$ is injective on torsion cycles of codimension $i \leq 2$ (cf. [15]).

The main result proved in this paper is the following interpretation of degree 4 unramified cohomology with finite coefficients:

Theorem 2.3. Assume that the group $H_{B}^{5}(X, \mathbb{Z}) / N^{2} H_{B}^{5}(X, \mathbb{Z})$ has no torsion. Then the quotient of $H_{n r}^{4}(X, \mathbb{Q} / \mathbb{Z})$ by $H_{n r}^{4}(X, \mathbb{Z}) \otimes \mathbb{Q} / \mathbb{Z}$ identifies to the group $\mathcal{T}^{3}(X)$.

Equivalently, there is an exact sequence

$$
0 \rightarrow H_{n r}^{4}(X, \mathbb{Z}) \otimes \mathbb{Q} / \mathbb{Z} \rightarrow H_{n r}^{4}(X, \mathbb{Q} / \mathbb{Z}) \rightarrow \operatorname{Tors}\left(C H^{3}(X) / a l g\right) \stackrel{c l_{\mathcal{D}, t r}^{3}}{\rightarrow} H_{\mathcal{D}}^{6}(X, \mathbb{Z}(3))_{t r} .
$$


Remark 2.4. The torsion part of the group $H_{B}^{5}(X, \mathbb{Z}) / N^{2} H_{B}^{5}(X, \mathbb{Z})$ is also a birational invariant of $X$. Looking again at what happens under a blow-up, this follows from the fact that the torsion part of the group $H_{B}^{3}(Y, \mathbb{Z})$ for $Y$ smooth and projective is of coniveau $\geq 1$, which is a consequence of the fact that the sheaf $\mathcal{H}_{Y}^{3}(\mathbb{Z})$ has no torsion by Theorem 1.4.

\section{Proof of the main result}

Proof of theorem 2.3. Recall the Bloch-Ogus formula (1.1) in section 1

$$
C H^{l+1}(X) / a l g=H^{l+1}\left(X_{Z a r}, \mathcal{H}^{l+1}(\mathbb{Z})\right) .
$$

Recall next the short exact sequence of sheaves on $X_{Z a r}$ from Corollary 1.5:

$$
0 \rightarrow \mathcal{H}^{l+1}(\mathbb{Z}) \rightarrow \mathcal{H}^{l+1}(\mathbb{Q}) \rightarrow \mathcal{H}^{l+1}(\mathbb{Q} / \mathbb{Z}) \rightarrow 0
$$

Combining the associated long exact sequence with (3.3), we get an exact sequence

$$
H^{l}\left(X_{Z a r}, \mathcal{H}^{l+1}(\mathbb{Q})\right) \rightarrow H^{l}\left(X_{Z a r}, \mathcal{H}^{l+1}(\mathbb{Q} / \mathbb{Z})\right) \stackrel{\gamma_{l}}{\rightarrow} \operatorname{Tors}\left(C H^{l+1}(X) / a l g\right) \rightarrow 0 .
$$

Let us now consider the Bloch-Ogus spectral sequence with coefficients in $\mathbb{Q} / \mathbb{Z}$. Taking into account the vanishing results (1.2) of section 1 , the deepest term of the associated filtration on $H^{2 l+1}\left(X_{Z a r}, \mathbb{Q} / \mathbb{Z}\right)$ is the term $E_{\infty}^{l, l+1}$ which is a sub-quotient of $E_{2}^{l, l+1}=H^{l}\left(X_{Z a r}, \mathcal{H}^{l+1}(\mathbb{Q} / \mathbb{Z})\right)$. But, still by the vanishing (1.2), no nonzero differential $d_{r}$ in this spectral sequence can start from $E_{r}^{l, l+1}, r \geq 2$, hence the graded piece $E_{\infty}^{l, l+1}$ is in fact a quotient of $E_{2}^{l, l+1}$. We denote by

$$
f_{l, \mathbb{Q} / \mathbb{Z}}: H^{l}\left(X_{Z a r}, \mathcal{H}^{l+1}(\mathbb{Q} / \mathbb{Z})\right) \rightarrow H_{B}^{2 l+1}(X, \mathbb{Q} / \mathbb{Z})
$$

the map which is obtained by composing the quotient map $E_{2}^{l, l+1} \rightarrow E_{\infty}^{l, l+1}$ and the inclusion $E_{\infty}^{l, l+1} \hookrightarrow H_{B}^{2 l+1}(X, \mathbb{Q} / \mathbb{Z})$.

The analysis of the Bloch-Ogus spectral sequence with coefficients in $\mathbb{Z}$ or $\mathbb{Q}$ produces in a similar way maps

$$
\begin{aligned}
f_{l, \mathbb{Q}}: H^{l}\left(X_{Z a r}, \mathcal{H}^{l+1}(\mathbb{Q})\right) & \rightarrow H_{B}^{2 l+1}(X, \mathbb{Q}), \\
f_{l, \mathbb{Z}}: H^{l}\left(X_{\text {Zar }}, \mathcal{H}^{l+1}(\mathbb{Z})\right) & \rightarrow H_{B}^{2 l+1}(X, \mathbb{Z}) .
\end{aligned}
$$

It is known (cf. [11]) that the image of $f_{l, \mathbb{Z}}$ is nothing but $N^{l} H_{B}^{2 l+1}(X, \mathbb{Z})$. We will denote

$$
\bar{f}_{l, \mathbb{Q}}: H^{l}\left(X, \mathcal{H}_{X}^{l+1}(\mathbb{Q})\right) \rightarrow \rightarrow H^{2 l+1}(X, \mathbb{Q} / \mathbb{Z})
$$

the map which is the composition of $f_{l, \mathbb{Q}}$ and of the natural map $H_{B}^{2 l+1}(X, \mathbb{Q}) \rightarrow H_{B}^{2 l+1}(X, \mathbb{Q} / \mathbb{Z})$. The exact sequence (3.5) thus provides us with a map

$$
c l_{l, t o r s, t r}: \text { Tors }\left(C H^{l}(X) / a l g\right) \cong \frac{H^{l}\left(X_{Z a r}, \mathcal{H}^{l+1}(\mathbb{Q} / \mathbb{Z})\right)}{\operatorname{Im}\left[H^{l}\left(X_{Z a r}, \mathcal{H}^{l+1}(\mathbb{Q})\right)\right]} \rightarrow \frac{H_{B}^{2 l+1}(X, \mathbb{Q} / \mathbb{Z})}{\operatorname{Im} \bar{f}_{l, \mathbb{Q}}},
$$

Let us now consider the case where $l=2$, so $2 l+1=5$. By the vanishing (1.2), the only nonzero differential $d_{r}$ which could arrive in $E_{r}^{2,3}$ is

$$
d_{2}^{0,4}: H^{0}\left(X_{Z a r}, \mathcal{H}^{4}(\mathbb{Q} / \mathbb{Z})\right) \rightarrow H^{2}\left(X_{Z a r}, \mathcal{H}^{3}(\mathbb{Q} / \mathbb{Z})\right) .
$$


Furthermore, we have

$$
\operatorname{Ker} d_{2}^{0,4}=\operatorname{Im}\left(H_{B}^{4}(X, \mathbb{Q} / \mathbb{Z}) \rightarrow H^{0}\left(X_{Z a r}, \mathcal{H}^{4}(\mathbb{Q} / \mathbb{Z})\right)\right)
$$

since, again by the vanishing (1.2), the higher $d_{r}$ 's starting from $E_{r}^{0,4}$ are 0 .

It follows that we have an exact sequence

$$
H_{B}^{4}(X, \mathbb{Q} / \mathbb{Z}) \rightarrow H^{0}\left(X_{Z a r}, \mathcal{H}^{4}(\mathbb{Q} / \mathbb{Z})\right) \stackrel{d_{2}}{\rightarrow} H^{2}\left(X_{Z a r}, \mathcal{H}^{3}(\mathbb{Q} / \mathbb{Z})\right) \rightarrow H_{B}^{5}(X, \mathbb{Q} / \mathbb{Z}),
$$

and similarly with coefficients $\mathbb{Z}$ and $\mathbb{Q}$. To summarize, we have the following commutative diagram where all lines and arrows are exact, the lines being induced by the short exact sequence $0 \rightarrow \mathbb{Z} \rightarrow \mathbb{Q} \rightarrow \mathbb{Q} / \mathbb{Z} \rightarrow 0$ of sheaves on $X_{c l}$ and the induced short exact sequences (3.4) on $X_{Z a r}$, and the columns being given by the exact sequences (3.8) with various coefficients :

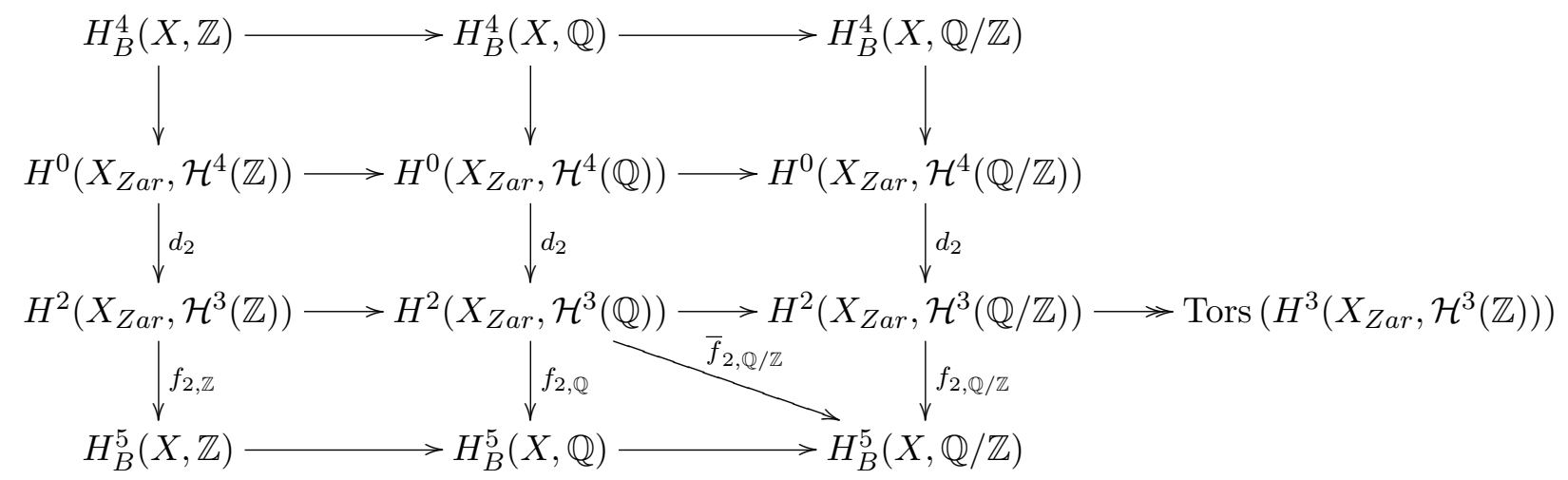

From this exact diagram, we deduce using the last column and (3.7) a surjective map

$$
H^{0}\left(X, \mathcal{H}^{4}(\mathbb{Q} / \mathbb{Z})\right) \rightarrow \operatorname{Ker}\left(c_{3, t o r s, t r}: \operatorname{Tors}\left(C H^{3}(X) / \operatorname{alg}\right) \rightarrow H_{B}^{5}(X, \mathbb{Q} / \mathbb{Z}) / \operatorname{Im} \bar{f}_{2, \mathbb{Q} / \mathbb{Z}}\right)
$$

whose kernel identifies to the quotient of the group

$$
\Gamma:=\left\{\alpha \in H^{0}\left(X_{Z a r}, \mathcal{H}^{4}(\mathbb{Q} / \mathbb{Z})\right), d_{2}(\alpha) \in \operatorname{Im}\left(H^{2}\left(X_{Z a r}, \mathcal{H}^{3}(\mathbb{Q})\right) \rightarrow H^{2}\left(X_{Z a r}, \mathcal{H}^{3}(\mathbb{Q} / \mathbb{Z})\right)\right\}\right.
$$

by the image of the group $H_{B}^{4}(X, \mathbb{Q} / \mathbb{Z})$.

Recall that we assumed that $H_{B}^{5}(X, \mathbb{Z}) / N^{2} H_{B}^{5}(X, \mathbb{Z})$ has no torsion. Let us show the following consequence:

Lemma 3.1. If $H_{B}^{5}(X, \mathbb{Z}) / N^{2} H_{B}^{5}(X, \mathbb{Z})$ has no torsion, the image of the natural map

$$
H_{B}^{4}(X, \mathbb{Q} / \mathbb{Z}) \rightarrow H^{0}\left(X_{Z a r}, \mathcal{H}^{4}(\mathbb{Q} / \mathbb{Z})\right)
$$

is contained in the image of the map

$$
H^{0}\left(X_{Z a r}, \mathcal{H}^{4}(\mathbb{Q})\right) \rightarrow H^{0}\left(X_{Z a r}, \mathcal{H}^{4}(\mathbb{Q} / \mathbb{Z})\right)
$$

Proof. Indeed, the analysis of the spectral sequence converging to $H^{5}\left(X_{c l}, \mathbb{Z}\right)=H_{B}^{5}(X, \mathbb{Z})$ shows that $H_{B}^{5}(X, \mathbb{Z}) / N^{2} H_{B}^{5}(X, \mathbb{Z})$ contains the subgroup $E_{\infty}^{1,4}=\operatorname{Ker} d_{2}$ of $H^{1}\left(X_{Z a r}, \mathcal{H}^{4}(\mathbb{Z})\right)$. Under our assumption,

$$
\operatorname{Ker}\left(d_{2}: H^{1}\left(X_{Z a r}, \mathcal{H}^{4}(\mathbb{Z})\right) \rightarrow H^{3}\left(X_{Z a r}, \mathcal{H}^{3}(\mathbb{Z})\right)\right),
$$


being contained in $H_{B}^{5}(X, \mathbb{Z}) / N^{2} H_{B}^{5}(X, \mathbb{Z})$, is without torsion. We deduce from this that the composite map

$$
H_{B}^{4}(X, \mathbb{Q} / \mathbb{Z}) \rightarrow H^{0}\left(X_{Z a r}, \mathcal{H}^{4}(\mathbb{Q} / \mathbb{Z})\right) \rightarrow H^{1}\left(X_{\text {Zar }}, \mathcal{H}^{4}(\mathbb{Z})\right)
$$

whose image is both of torsion and contained in Ker $d_{2}$, is identically zero, which implies the result by the exact sequence

$$
H^{0}\left(X_{Z a r}, \mathcal{H}^{4}(\mathbb{Q})\right) \rightarrow H^{0}\left(X_{Z a r}, \mathcal{H}^{4}(\mathbb{Q} / \mathbb{Z})\right) \rightarrow H^{1}\left(X_{Z a r}, \mathcal{H}^{4}(\mathbb{Z})\right)
$$

We prove now:

Lemma 3.2. The group $\Gamma$ of (3.11) identifies to the image of $H^{0}\left(X_{Z a r}, \mathcal{H}^{4}(\mathbb{Q})\right)$ in $H^{0}\left(X_{Z a r}, \mathcal{H}^{4}(\mathbb{Q} / \mathbb{Z})\right)$.

Proof. This is diagram chasing in the diagram (3.9). Let $\alpha \in \Gamma \subset H^{0}\left(X_{Z a r}, \mathcal{H}^{4}(\mathbb{Q} / \mathbb{Z})\right)$, so that by definition $d_{2} \alpha=\bar{\beta}$ for some $\beta \in H^{2}\left(X_{Z a r}, \mathcal{H}^{3}(\mathbb{Q})\right)$. The class $\bar{\beta}$ is annihilated by $f_{2, \mathbb{Q} / \mathbb{Z}}$ and thus, the image of the class $\beta$ under the map $f_{2, \mathbb{Q}}$ is an element of $H_{B}^{5}(X, \mathbb{Q})$ which vanishes in $H_{B}^{5}(X, \mathbb{Q} / \mathbb{Z})$. We thus have

$$
f_{2, \mathbb{Q}}(\beta)=\operatorname{Im} \gamma,
$$

for some $\gamma \in H_{B}^{5}(X, \mathbb{Z})$, which is clearly of torsion in $H_{B}^{5}(X, \mathbb{Z}) / \operatorname{Im} f_{2, \mathbb{Z}}=H_{B}^{5}(X, \mathbb{Z}) / N^{2} H_{B}^{5}(X, \mathbb{Z})$.

But by assumption the group $H_{B}^{5}(X, \mathbb{Z}) / N^{2} H_{B}^{5}(X, \mathbb{Z})$ has no torsion; hence there exists $\beta^{\prime \prime} \in H^{2}\left(X_{Z a r}, \mathcal{H}^{3}(\mathbb{Z})\right)$ such that $f_{2, \mathbb{Z}}\left(\beta^{\prime \prime}\right)-f_{2, \mathbb{Q}}(\beta)=0$ in $H_{B}^{5}(X, \mathbb{Q})$. The second vertical exact sequence in the diagram (3.9) then shows that $f_{2, \mathbb{Z}}\left(\beta^{\prime \prime}\right)-f_{2, \mathbb{Q}}(\beta)=d_{2}(\delta)$ for some $\delta \in$ $H^{0}\left(X_{Z a r}, \mathcal{H}^{4}(\mathbb{Q})\right)$. Denoting by $\bar{\delta}$ the image of $\delta$ in $H^{0}\left(X_{Z a r}, \mathcal{H}^{4}(\mathbb{Q} / \mathbb{Z})\right)$, the class $\alpha-\bar{\delta}$ is then annihilated by $d_{2}: H^{0}\left(X_{Z a r}, \mathcal{H}^{4}(\mathbb{Q} / \mathbb{Z})\right) \rightarrow H^{2}\left(X_{Z a r}, \mathcal{H}^{3}(\mathbb{Q} / \mathbb{Z})\right)$ and thus comes from $H_{B}^{4}(X, \mathbb{Q} / \mathbb{Z})$ as it follows from the third vertical exact sequence of the diagram (3.9). It then also comes from an element of $H^{0}\left(X_{Z a r}, \mathcal{H}^{4}(\mathbb{Q})\right)$ by Lemma 3.1.

From (3.10) and the above description of $\Gamma$, we thus established, under the assumption that $H^{5}(X, \mathbb{Z}) / N^{2} H^{5}(X, \mathbb{Z})$ is without torsion, the following exact sequence

$$
H_{n r}^{4}(X, \mathbb{Z}) \otimes \mathbb{Q} / \mathbb{Z} \rightarrow H_{n r}^{4}(X, \mathbb{Q} / \mathbb{Z}) \rightarrow \operatorname{Ker}_{3} l_{3, \text { tors }, t r} \rightarrow 0 .
$$

The proof of Theorem 2.3 is then concluded using Proposition 3.4 below, which implies that we have the equality

$$
\operatorname{Ker} c l_{3, t o r s, t r}=\operatorname{Ker}\left(c l_{\mathcal{D}, t r \mid \operatorname{Tors}\left(C H^{3}(X) / \text { alg }\right)}^{3}\right)=: \mathcal{T}^{3}(X) \text {. }
$$

In order to state Proposition 3.4, we will need the following Lemma.

Lemma 3.3. For any smooth complex projective variety $X$, there is a canonical isomorphism

$$
\iota_{l}: \operatorname{Tors}\left(H_{\mathcal{D}}^{2 l+2}(X, \mathbb{Z}(l+1))_{t r}\right) \cong \frac{H_{B}^{2 l+1}(X, \mathbb{Q} / \mathbb{Z})}{\operatorname{Im} \bar{f}_{l, \mathbb{Q} / \mathbb{Z}}},
$$

where the map $\bar{f}_{l, \mathbb{Q} / \mathbb{Z}}$ was introduced in (3.6). 
Proof. We have an exact sequence

$$
0 \rightarrow J^{2 l+1}(X)_{a l g} \rightarrow H_{\mathcal{D}}^{2 l+2}(X, \mathbb{Z}(l+1)) \rightarrow H_{\mathcal{D}}^{2 l+2}(X, \mathbb{Z}(l+1))_{t r} \rightarrow 0,
$$

where

$$
J^{2 l+1}(X)_{a l g}=\left\langle\tau_{W *} J^{1}(\widetilde{W}), \operatorname{codim} W=l\right\rangle
$$

is the algebraic part of the intermediate Jacobian $J^{2 l+1}(X)$. The group $J^{2 l+1}(X)_{\text {alg }}$ being divisible, (3.13) induces an exact sequence of torsion subgroups:

$$
0 \rightarrow \operatorname{Tors}\left(J^{2 l+1}(X)_{a l g}\right) \rightarrow \operatorname{Tors}\left(H_{\mathcal{D}}^{2 l+2}(X, \mathbb{Z}(l+1))\right) \rightarrow \operatorname{Tors}\left(H_{\mathcal{D}}^{2 l+2}(X, \mathbb{Z}(l+1))_{t r}\right) \rightarrow 0 .
$$

The torsion subgroup of $H_{\mathcal{D}}^{2 l+2}(X, \mathbb{Z}(l+1))$ is easy to analyze : indeed,we have the exact sequence of complexes of sheaves on $X_{c l}$

$$
0 \rightarrow \mathbb{Z}_{\mathcal{D}}(l+1) \rightarrow \mathbb{Q}_{\mathcal{D}}(l+1) \rightarrow \mathbb{Q} / \mathbb{Z} \rightarrow 0,
$$

where $\mathbb{Z}_{\mathcal{D}}(l+1)$ is the Deligne complex $0 \rightarrow \mathbb{Z} \rightarrow \mathcal{O}_{X} \rightarrow \ldots \rightarrow \Omega_{X}^{l} \rightarrow 0$, where $\mathbb{Z}$ is put in degree 0 , which satisfies by definition $\mathbb{H}^{2 l+2}\left(X_{c l}, \mathbb{Z}_{\mathcal{D}}(l+1)\right)=H_{\mathcal{D}}^{2 l+2}(X, \mathbb{Z}(l+1))$, and $\mathbb{Q}_{\mathcal{D}}(l+1)$ is defined similarly, replacing $\mathbb{Z}$ by $\mathbb{Q}$.

The exact sequence (3.15) of complexes of sheaves on $X_{c l}$ induces by taking cohomology the exact sequence

$$
\mathbb{H}^{2 l+1}\left(X, \mathbb{Q}_{\mathcal{D}}(l+1)\right) \rightarrow H_{B}^{2 l+1}(X, \mathbb{Q} / \mathbb{Z}) \rightarrow \operatorname{Tors}\left(H_{\mathcal{D}}^{2 l+2}(X, \mathbb{Z}(l+1))\right) \rightarrow 0 .
$$

But, as $X$ is projective and smooth, one immediately sees, using the exact sequence of complexes

$$
0 \rightarrow \Omega_{X_{a n}}^{\bullet \leq l}[1] \rightarrow \mathbb{Q}_{\mathcal{D}}(l+1) \rightarrow \mathbb{Q} \rightarrow 0
$$

that the map

$$
\mathbb{H}^{2 l+1}\left(X, \mathbb{Q}_{\mathcal{D}}(l+1)\right) \rightarrow \mathbb{H}_{B}^{2 l+1}(X, \mathbb{Q})
$$

is zero. Indeed, the long exact sequence associated to (3.17) writes

$$
\mathbb{H}^{2 l+1}\left(X, \mathbb{Q}_{\mathcal{D}}(l+1)\right) \rightarrow \mathbb{H}_{B}^{2 l+1}(X, \mathbb{Q}) \rightarrow \mathbb{H}^{2 l+1}\left(X, \Omega_{X_{a n}}^{\bullet<l}\right),
$$

where the last term is equal by $[23, \mathrm{I}, 8.3 .3]$ to $H_{B}^{2 l+1}(X, \mathbb{C}) / F^{l+1} H_{B}^{2 l+1}(X, \mathbb{C})$, where $F^{i}$ stands for the Hodge filtration. Now, by Hodge symmetry, the $\operatorname{map} H_{B}^{2 l+1}(X, \mathbb{Q}) \rightarrow H_{B}^{2 l+1}(X, \mathbb{C}) / F^{l+1} H_{B}^{2 l+1}(X, \mathbb{C})$ is injective.

A fortiori the map

$$
\mathbb{H}^{2 l+1}\left(X, \mathbb{Q}_{\mathcal{D}}(l+1)\right) \rightarrow \mathbb{H}_{B}^{2 l+1}(X, \mathbb{Q} / \mathbb{Z})
$$

is zero. We thus get from $(3.16)$ an isomorphism $H^{2 l+1}(X, \mathbb{Q} / \mathbb{Z}) \cong \operatorname{Tors}\left(H_{\mathcal{D}}^{2 l+2}(X, \mathbb{Z}(l+1))\right)$.

For any irreducible subvariety $W \subset X$ of codimension $l$, with desingularization $\tau_{W}: \widetilde{W} \rightarrow$ $X$, the description of $\operatorname{Pic}^{0}(\widetilde{W})$ as the torus $J^{1}(\widetilde{W})$ identifies Tors $\left(\operatorname{Pic}^{0}(\widetilde{W})\right)$ to the image of $H^{1}(\widetilde{W}, \mathbb{Q})$ in $H^{1}(\widetilde{W}, \mathbb{Q} / \mathbb{Z})$. It follows that

$$
\operatorname{Tors}\left(J^{2 l+1}(X)_{a l g}\right)=\left\langle\operatorname{Im}\left(\tau_{W *}: H^{1}(\widetilde{W}, \mathbb{Q}) \rightarrow H^{2 l+1}(X, \mathbb{Q} / \mathbb{Z})\right)\right\rangle,
$$

which implies the desired result since we also have

$$
\left\langle\operatorname{Im}\left(\tau_{W *}: H^{1}(\widetilde{W}, \mathbb{Q}) \rightarrow H^{2 l+1}(X, \mathbb{Q} / \mathbb{Z})\right)\right\rangle=\operatorname{Im} \bar{f}_{l, \mathbb{Q} / \mathbb{Z}}
$$

by definition of $\bar{f}_{l, \mathbb{Q} / \mathbb{Z}}($ cf. $(3.6))$. 
We now have the following interpretation of the maps $c l_{l, t o r s, t r}$ :

Proposition 3.4. The map $\mathrm{cl}_{l, t o r s, t r}$ of (3.7) identifies via the isomorphism $\iota_{l}$ of (3.12) to the restriction of $c l_{\mathcal{D}, t r}^{l+1}$ to the torsion subgroup Tors $\left(\mathrm{CH}^{l+1}(X) / \mathrm{alg}\right)$, with value in the torsion subgroup of $H_{\mathcal{D}}^{2 l+2}(X, \mathbb{Z}(l+1))_{t r}$.

Proof. Looking at the arguments given in the beginning of the proof of Theorem 2.3, it suffices to show that for any $\beta \in H^{l}\left(X, \mathcal{H}^{l+1}(\mathbb{Q} / \mathbb{Z})\right)$, we have:

$$
f_{l, \mathbb{Q} / \mathbb{Z}}(\beta) \bmod \operatorname{Im} \bar{f}_{l, \mathbb{Q} / \mathbb{Z}}=\iota_{l}\left(c l_{\mathcal{D}, t r}^{l+1}\left(\gamma_{l}(\beta)\right)\right),
$$

where $\gamma_{l}: H^{l}\left(X, \mathcal{H}^{l+1}(\mathbb{Q} / \mathbb{Z})\right) \rightarrow \operatorname{Tors}\left(C H^{l+1}(X) /\right.$ alg $)$ was introduced in the exact sequence $(3.5)$.

The equality (3.18) can be shown in the following way using the construction of the Deligne cycle class map involving the Cheeger-Simons differential characters (cf. [23, I,12.3.3]).

The class $\beta$ is represented according to the results of section 1 (the Bloch-Ogus resolution of the sheaf $\mathcal{H}^{l+1}(\mathbb{Q} / \mathbb{Z})$ ) by the data of subvarieties $W_{i}$ of $X$ of codimension $l$, of smooth Zariski open sets $W_{i}^{0} \subset W_{i}$, and of classes $\beta_{i} \in H^{1}\left(W_{i}^{0}, \mathbb{Q}\right)$ satisfying the condition:

$$
\sum_{i} \partial \beta_{i}=0 \text { in } \mathcal{Z}^{l+1}(X) \otimes \mathbb{Q} / \mathbb{Z}
$$

where $\partial$ is the last differential of the Bloch-Ogus resolution (cf. Theorem 1.1) of $\mathcal{H}^{l+1}(\mathbb{Q} / \mathbb{Z}$ ).

Let $W=\cup_{i} W_{i}, \partial W_{i}:=W_{i} \backslash W_{i}^{0}$ and $\partial W:=\cup_{i} \partial W_{i}$. We can assume that the $W_{i}^{0}$ are disjoint, and then the equality (3.19) allows to describe the classes $\gamma_{l}(\beta)$ and $f_{l, \mathbb{Q} / \mathbb{Z}}(\beta)$ in the following way. First of all we have a cycle $Z^{\prime}:=\sum_{i} \partial \beta_{i} \in \mathcal{Z}^{l+1}(X)$ with integral coefficients which clearly represents $\gamma_{l}(\beta)$, because $\gamma_{l}$ is nothing but the connecting map associated to the short exact sequence (3.4).

On the other hand, the classes $\beta_{i}$ on $W_{i}^{0}$ give by Poincaré duality relative homology classes $B_{i} \in H_{2 d-2 l-1, B}\left(W_{i}, \partial W_{i}, \mathbb{Q}\right), d:=\operatorname{dim} \mathrm{X}$ which have the property that the sum of their boundaries vanishes in $H_{2 d-2 l-2, B}(\partial W, \mathbb{Q} / \mathbb{Z})$. Hence the data of the $B_{i}$ 's determine a homology class in $H_{2 d-2 l-1, B}(W, \mathbb{Q} / \mathbb{Z})$, which provides via the inclusion of $W$ in $X$ a class

$$
B \in H_{2 d-2 l-1, B}(X, \mathbb{Q} / \mathbb{Z}) \cong H_{B}^{2 l+1}(X, \mathbb{Q} / \mathbb{Z}),
$$

where the last isomorphism is given by Poincaré duality on $X$. A look at the construction of the Gersten type resolution by Bloch-Ogus shows that $B$ is in fact equal to $f_{l, \mathbb{Q} / \mathbb{Z}}(\beta)$.

It thus remains only to show that $c l_{\mathcal{D}}\left(Z^{\prime}\right)=B$ in $H_{B}^{2 l+1}(X, \mathbb{Q} / \mathbb{Z}) \cong \operatorname{Tors}\left(H_{\mathcal{D}}^{2 l+2}(X, \mathbb{Z}(l+1))\right)$. One has an inclusion

$$
H_{B}^{2 l+1}(X, \mathbb{Q} / \mathbb{Z}) \hookrightarrow \operatorname{Hom}\left(H_{2 d-2 l-1, B}(X, \mathbb{Z}), \mathbb{Q} / \mathbb{Z}\right) .
$$

According to $[23, \mathrm{I}, 12.3 .2]$, a Deligne cohomology class $e \in H_{D}^{2 l+2}(X, \mathbb{Z}(l+1))$ is represented by a homomorphism $\mathcal{C}_{2 l+1, c} \rightarrow \mathbb{R} / \mathbb{Z}$, (which takes values in $\mathbb{Q} / \mathbb{Z}$ if $e$ is of torsion,) where $\mathcal{C}_{2 l+1, c}$ is the group of closed integral singular chains of dimension $2 l+1$ of $X_{c l}$. Given a cycle $z$ of $X$ of dimension $d-l-1$ whose rational cohomology class $[z]$ vanishes in $H_{B}^{2 l+2}(X, \mathbb{Q})$, one constructs such a representative $\phi_{z}: \mathcal{C}_{2 l+1, c} \rightarrow \mathbb{R} / \mathbb{Z}$ for $c l_{D}(z)$ in the following way (cf. [23, I,12.3.3]): one chooses a differential form $\phi$ of type $(l+1, l)$ on $X$ with singularities (of prescribed type) only along $\operatorname{Supp} z$, such that the following equality of currents holds:

$$
\int_{z}=d \phi
$$


For any closed chain $\Gamma$ of dimension $2 l+1$ of $X$, one writes

$$
\Gamma=\Gamma^{\prime}+\partial T
$$

where $T$ is a chain of dimension $2 l+2$ of $X$ and $\Gamma^{\prime}$ is another chain of dimension $2 l+1$ of $X$ not intersecting $\operatorname{Supp} z$. One then sets

$$
\phi_{z}(\Gamma)=\int_{\Gamma^{\prime}} \phi
$$

where the value of the right hand side modulo $\mathbb{Z}$ is easily shown not to depend on $\Gamma^{\prime}, T$.

Coming back to the cycle $Z^{\prime}$ introduced above, up to modifying the $\beta_{i}$ 's by classes coming from $H^{1}\left(\widetilde{W}_{i}, \mathbb{Q}\right)$ (which won't change the result after passing to the transcendental part of the Deligne cycle class), one can choose 1 -forms $\tilde{\beta}_{i}$ on $W_{i}^{0}$ representing the $\beta_{i}$ 's, with logarithmic singularities along $\partial W_{i}$, and then one can take for $\phi$ a form of the following type :

$$
\phi=\sum_{i} j_{i *} \tilde{\beta}_{i}
$$

where $j_{i *} \tilde{\beta}_{i}$ is a singular form obtained by extending $\tilde{\beta}_{i}$ to a closed form defined in a neighborhood $T_{\epsilon} W_{i}$ of $W_{i}$, singular along $\partial W_{i}$, and by taking its cup-produit with a compactly supported form in this neighborhood, representing along $W_{i}^{0}$ the Thom class of $\left(T_{\epsilon} W_{i}, \partial T_{\epsilon} W_{i}\right)$. It then only remains to show that one has for any closed chain $\Gamma$ not meeting this neighborhood of $\partial W_{i}$ and meeting transversally the $W_{i}^{0}$ s:

$$
\langle B, \Gamma\rangle=\int_{\Gamma} \phi \bmod . \mathbb{Z},
$$

where the contraction on the left is induced by (3.20). But by the construction of $\phi$ and by the fact that $\Gamma$ does not meet the locus $\partial W_{i}$ where $\beta_{i}$ is singular, the term on the right is equal to

$$
\sum_{i} \int_{\Gamma \cap W_{i}} \tilde{\beta}_{i} \in \mathbb{Q}
$$

while the term on the left is equal to

$$
\sum_{i} \sharp\left(\Gamma \cap B_{i}\right)=\sum_{i} \sharp\left(\Gamma \cap W_{i} \cap B_{i}\right) \in \mathbb{Q},
$$

where $\sharp$ is the sum of the local intersection numbers (with coefficients in $\mathbb{Q}$, as the $B_{i}$ 's) counted with the signs given by the orientations, these local intersection numbers being well-defined as each $\Gamma \cap W_{i}$ is supported in $W_{i}^{0}$.

As $\tilde{\beta}_{i}$ represents $\beta_{i} \in H_{B}^{1}\left(W_{i}^{0}, \mathbb{Q}\right)$, and the closed 1-chain $\Gamma \cap W_{i}$ is supported on $W_{i}^{0}$, the equality of (3.22) and (3.23) in $\mathbb{Q} / \mathbb{Z}$ is simply obtained as the sum of equalities of intersection numbers between $H_{1, B}\left(W_{i}^{0}, \mathbb{Z}\right)$ and $H_{B}^{1}\left(W_{i}, \partial W_{i}, \mathbb{Q}\right)$.

\section{Discussion of geometric examples}

There are two interesting groups involved in Theorem 0.2 , and especially its corollary 0.3 , namely unramified cohomology with torsion coefficients $H_{n r}^{4}(X, \mathbb{Q} / \mathbb{Z})$ and the subgroup $\mathcal{T}^{3}(X)$ of the torsion group of the Griffiths group which is annihilated by the "transcendental part" of the Deligne cycle class. They are isomorphic under two conditions : $C H_{0}(X)$ is supported on a three 
dimensional subvariety, and (this is the second assumption in Corollary 0.3) there is no torsion in $H^{5}(X, \mathbb{Z}) / N^{2} H^{5}(X, \mathbb{Z})$. We will discuss below examples showing the non triviality of one of these two groups. As we will see, it is hard to guarantee also that the second assumption in Corollary 0.3 is satisfied, in order that the non-vanishing of one group implies the non-vanishing of the other one.

Remark 4.1. For certain types of varieties, the absence of torsion in $H^{5}(X, \mathbb{Z}) / N^{2} H^{5}(X, \mathbb{Z})$ is easy to check: for example those for which $H^{5}(X, \mathbb{Z})=0$ (eg a general complete intersection of dimension $>5$ of ample hypersurfaces in a smooth projective variety $Y$ also satisfying $\left.H^{5}(Y, \mathbb{Z})=0\right)$. If one considers a general complete intersection $X$ of dimension 5 of ample hypersurfaces in a variety $Y$ satisfying $H^{5}(Y, \mathbb{Z})=0$, then it is known by the Lefschetz theorem on hyperplane sections that $H^{5}(X, \mathbb{Z})$ has no torsion. To guarantee that the quotient $H^{5}(X, \mathbb{Z}) / N^{2} H^{5}(X, \mathbb{Z})$ has no torsion, it suffices of course that $N^{2} H^{5}(X, \mathbb{Z})=0$, which can be done usually by monodromy considerations. But it is not easy to construct examples of this type which have a non trivial $\mathcal{T}^{3}(X)$ or $H_{n r}^{4}(X, \mathbb{Q} / \mathbb{Z})$ group.

The article [16] provided the first known examples of cycles which are non trivial modulo algebraic equivalence but are annihilated by the Deligne cycle class. In fact, the first non trivial elements in the Griffiths group of cycles homologous to zero modulo algebraic equivalence had been found by Griffiths [12] and proved to be non algebraically equivalent to zero using their Abel-Jacobi invariants. Later on, Schoen [18] produced torsion elements in the Griffiths group, but still used the Abel-Jacobi map to prove that they are non zero.

Nori's examples in [16] do not provide torsion elements in the Griffiths group. They are obtained by restricting a cycle non homologous to zero but with primitive class (for example the difference $Z$ of two rulings in an even dimensional quadric of dimension $\geq 6$ ) to a very general complete intersection of at least two hypersurfaces of sufficiently large degree. If we start from a quadric $Q$ of dimension 6 , and take for $X$ a very general complete intersection of two high degree hypersurfaces in it, Nori's theorem says that the cycle $Z_{\mid X}$ is non trivial in $\operatorname{Griff}^{3}(X)$, and it is of course annihilated by the Abel-Jacobi map, since the corresponding intermediate Jacobian $J^{5}(X)$ is trivial. This cycle is obtained as $Z_{\mid X}$ where $Z$ is the difference of the two rulings of $Q$. A crucial point is Nori's proof is the fact that the class of $Z$ in $H^{6}(X, \mathbb{Q})$ is nonzero. One could of course in the above construction start from an ambient sixfold carrying a torsion cycle $Z$ with nonzero cohomology class, but it is unlikely that Nori's connectivity theorem, which concerns cohomology with rational coefficients and is used in the proof of the nontriviality of $Z_{\mid X}$ in the previous situation, is true for integral coefficients. Note also that in Nori's theorem, the complete intersections $X$ have to be ample enough, so that they do not satisfy the condition that $C H_{0}(X)$ is supported on a threefold.

Concerning unramified cohomology with torsion coefficients, Peyre constructs in [17] examples of unirational varieties $X$ (for example over $K=\overline{\mathbb{Q}}$ ) with nonzero group $H_{n r}^{4}(X, \mathbb{Q} / \mathbb{Z}$ ). Unfortunately, while the construction of the function field $K(X)$ is explicit, the smooth projective models $X$ are not easy to describe, and it is not clear whether they satisfy our assumption that $H_{B}^{5}(X, \mathbb{Z}) / N^{2} H_{B}^{5}(X, \mathbb{Z})$ is nonzero. So we cannot use Corollary 0.3 to conclude that these $X$ 's have some torsion element in $\operatorname{Griff}^{3}(X)$ which is annihilated by the transcendental cycle class $c l_{\mathcal{D}, t r}^{3}$.

In the paper [20], torsion codimension 3 cycles which are not algebraically equivalent to 0 and are annihilated by the Abel-Jacobi map are constructed as follows. Let $p \geq 5$ be a prime integer. Let $X$ be a hypersurface of degree $p^{3}$ in $\mathbb{P}^{4}$. We assume that $X$ contains a rational curve $C$ of degree $p$, and is very general otherwise. Let $S$ be a surface which admits a copy of $\mathbb{Z} / p \mathbb{Z}$ as a direct summand in $H^{2}(S, \mathbb{Z})$. Let $c$ be a generator of the torsion of $H^{2}(S, \mathbb{Z})$ and 
write $\tilde{c}:=c_{1}(\mathcal{L}) \in \operatorname{Pic} S$, where $\mathcal{L}$ is a $p$-torsion line bundle on $S$.

Let $W=S \times X$ and consider the codimension 3 cycle

$$
p r_{1}^{*} \tilde{c} \cdot p r_{2}^{*} C
$$

on $W$. It is clearly of torsion as $\tilde{c}$ is, and it is annihilated by the Abel-Jacobi map because the Deligne cycle class of $\tilde{c}$ is of $p$-torsion, while the Deligne cycle class of $C$ is divisible be $p$.

We have now:

Theorem 4.2. (Soulé-Voisin [20]) For a very general $X$ containing $C$, the cycle $p_{1}^{*} \tilde{c} \cdot \operatorname{pr}_{2}^{*} C$ is not divisible by $p$ in $C H^{3}(W)$, and in particular is not algebraically equivalent to 0.

This example however does not necessarily correspond to a non zero element in $H_{n r}^{4}(W, \mathbb{Q} / \mathbb{Z})$ because the group $H^{5}(W, \mathbb{Z}) / N^{2} H^{5}(W, \mathbb{Z})$ has some $p$-torsion in this case.

Our final goal is to exhibit an example of smooth projective variety $W$ for which the group $\mathcal{T}^{3}(W)$ is non zero, the group $C H_{0}(W)$ is supported on a subvariety of dimension $\leq 3$, and which also satisfies the property that $H^{5}(W, \mathbb{Z}) / N^{2} H^{5}(W, \mathbb{Z})$ has no torsion. (In fact we only have "potential" examples, as the proof that $H^{5}(W, \mathbb{Z}) / N^{2} H^{5}(W, \mathbb{Z})$ has no torsion depends on the generalized Hodge conjecture [13].) For these varieties, we will conclude (again under GHC) that $\mathcal{T}^{3}(W) \cong H_{n r}^{4}(W, \mathbb{Q} / \mathbb{Z}) \neq 0$ by Corollary 0.3 .

We will apply for this a product construction similar to the one described above with $X$ replaced by the threefolds $Y$ constructed in [8, Section 5.7].

These examples differ from Kollár's example by the fact that they satisfy $H^{3}\left(Y, \mathcal{O}_{Y}\right)=0$, and thus their degree 3 cohomology is conjecturally of coniveau 1 by the generalized Hodge conjecture (cf. [13]), although we were not able to prove this.

The examples are constructed as follows: Let $G=\mathbb{Z} / 5 \mathbb{Z}$. Let us choose a non trivial 5th root $\zeta$ of the unity and a generator $g$ of $G$, and let $G$ act on $\mathbb{P}_{x y}^{1}=\operatorname{Proj} \mathbb{C}[x, y]$ and on $\mathbb{P}^{3}=\operatorname{Proj} \mathbb{C}\left[x_{0}, x_{1}, x_{2}, x_{3}\right]$ in the following way:

$$
\begin{gathered}
g^{*} x=x, g^{*} y=\zeta y, \\
g^{*} x_{i}=\zeta^{i} x_{i}, i=0, \ldots, 3 .
\end{gathered}
$$

Let $X \subset \mathbb{P}^{1} \times \mathbb{P}^{3}$ be an hypersurface of bidegree $(3,4)$ defined by an equation $f=0$, where $f \in H^{0}\left(\mathbb{P}^{1} \times \mathbb{P}^{3}, \mathcal{O}_{\mathbb{P}^{1} \times \mathbb{P}^{3}}(3,4)\right)$ is invariant under $G$. Such an $X$ is not smooth but has ordinary quadratic singularities at the fixed points of the action. Let $Y$ be a desingularization of $X / G$. The following result is proved in [8]:

Proposition 4.3. Let $X, Y$ be as above. If $X$ is very general, then :

(i) One has the vanishing $H^{i}\left(Y, \mathcal{O}_{Y}\right)=0$ for $i>0$.

(ii) The group $Z^{4}(Y):=H d g^{4}(Y, \mathbb{Z}) /<[Z], Z \subset Y, \operatorname{dim} Z=1>$ is nonzero.

The proof of (ii) involves the following specialization $X_{0}$ of $X$ (similar to those introduced first by Starr in [21]): Consider the $G$-equivariant degree 4 morphism

$$
\phi: \mathbb{P}_{u v}^{1} \rightarrow \mathbb{P}_{x y}^{1}
$$

defined by $\phi^{*} x=u^{4}, \phi^{*} y=v^{4}$, where $u, v$ are homogeneous coordinates on $\mathbb{P}^{1}$, with the following linearized action of $G: g^{*} u=u, g^{*} v=\zeta^{4} v$. We choose a generic $G$-invariant section $Q$ of $H^{0}\left(\mathbb{P}^{1} \times \mathbb{P}^{3}, \mathcal{O}_{\mathbb{P}^{1} \times \mathbb{P}^{3}}(3,1)\right)$.

Such a $Q$ has the following form:

$$
Q=u^{3} x_{0}+u^{2} v x_{1}+u v^{2} x_{2}+v^{3} x_{3} .
$$


Let $\Gamma \subset \mathbb{P}^{1} \times \mathbb{P}^{3}$ be the divisor of $Q$, and let $X_{0}:=(\phi, I d)(\Gamma)$. As the degree of $\phi$ is $4, X_{0}$ is a member of the linear system $\left|\mathcal{O}_{\mathbb{P}^{1} \times \mathbb{P}^{3}}(3,4)\right|$. Furthermore $X_{0}$ is defined by a $G$-invariant equation.

The non normal locus $\Sigma$ of $X_{0}$ is the following surface : Write $\Gamma$ as $\cup_{t \in \mathbb{P}^{1}} H_{t}$ where $H_{t} \subset \mathbb{P}^{3}$ is an hyperplane. Then if

$$
\Sigma^{\prime}:=\cup_{t \neq t^{\prime}, \phi(t)=\phi\left(t^{\prime}\right)} H_{t} \cap H_{t^{\prime}}
$$

(or rather its Zariski closure in $\Gamma$, as the above locus is not closed) we have $\Sigma=(\phi, I d)\left(\Sigma^{\prime}\right) \subset X_{0}$. As $\phi: \mathbb{P}_{u v}^{1} \rightarrow \mathbb{P}_{x y}^{1}$ is a Galois cover of group $\mathbb{Z} / 4 \mathbb{Z}$, the above locus $\Sigma^{\prime}$ has two components $\Sigma_{0}^{\prime}$ and $\Sigma_{1}^{\prime}$ : let $\sigma$ be the unique element of order 2 in $\mathbb{Z} / 4 \mathbb{Z}$. Then

$$
\begin{gathered}
\Sigma_{0}^{\prime}=\cup_{t \in \mathbb{P}_{u v}^{1}} H_{t} \cap H_{\sigma(t)} \\
\Sigma_{1}^{\prime}=\cup_{t \in \mathbb{P}_{u v}^{1}, g \neq \sigma, I d} H_{t} \cap H_{g(t)} .
\end{gathered}
$$

Both $\Sigma_{0}^{\prime}$ and $\Sigma_{1}^{\prime}$ map via a morphism of degree 2 to their image $\Sigma_{0}$, resp. $\Sigma_{1}$ in $X_{0}$. The surface $\Sigma_{0}^{\prime} \subset \mathbb{P}_{u v}^{1} \times \mathbb{P}^{3}$ is a $\mathbb{P}^{1}$-bundle over $\mathbb{P}_{u v}^{1}$ which is invariant under the involution $(\sigma, I d)$. Consider a section of this $\mathbb{P}^{1}$-bundle which is invariant under $(\sigma, I d)$, for example the intersection of $\Sigma_{0}^{\prime}$ with the inverse image $p_{2}^{-1}(P)$ where $P \subset \mathbb{P}^{3}$ is a plane.

The image of this curve in $\Sigma_{0}$ is thus a curve $C_{0} \subset X_{0}$ which has degree 2 over $\mathbb{P}_{x y}^{1}$.

Choose now $C_{0}$ as before (where the plane $P$ is generic), and let $X$ be a general $G$-invariant deformation of $X_{0}$ containing $C_{0}$. One checks that the singularities of $X$ are generic. It follows that if $Y$ is a desingularization of $X / G, Y$ satisfies as in Proposition 4.3 the vanishing condition $H^{i}\left(Y, \mathcal{O}_{Y}\right)=0$ for $i>0$. In particular $H^{3}\left(Y, \mathcal{O}_{Y}\right)=0$, so the Hodge structure on $H^{3}(Y, \mathbb{Q})$ has coniveau 1 and the generalized Hodge conjecture [13] predicts that $H^{3}(Y, \mathbb{Q})$ is supported on a proper closed algebraic subset of $Y$.

Let $S$ be a smooth projective surface satisfying $C H_{0}(S)=\mathbb{Z}$ and $\operatorname{Tors}\left(H_{B}^{2}(S, \mathbb{Z})\right)=\mathbb{Z} / 2 \mathbb{Z}$. For example, we can take for $S$ an Enriques surface. Let

$$
W=Y \times S .
$$

Since $C H_{0}(S)$ is supported on a point $s \in S, C H_{0}(W)$ is supported on the threefold $s \times Y \subset W$.

Proposition 4.4. Assume the generalized Hodge conjecture is satisfied by the coniveau 1 Hodge structure $H^{3}(Y, \mathbb{Q})$. Then $W$ satisfies the property that $H^{5}(W, \mathbb{Z}) / N^{2} H^{5}(W, \mathbb{Z})$ has no torsion. Furthermore, if $X$ is very general as above,

$$
\mathcal{T}^{3}(W) \cong H_{n r}^{4}(W, \mathbb{Q} / \mathbb{Z}) \neq 0 .
$$

Proof. We first check that $H_{B}^{5}(W, \mathbb{Z}) / N^{2} H_{B}^{5}(W, \mathbb{Z})$ has no torsion. It suffices for this to show that $H_{B}^{5}(W, \mathbb{Z}) / N^{2} H_{B}^{5}(W, \mathbb{Z})=0$. Recall that $Y$ is a desingularization of a quotient of $X$ by $G$ which is a group of order 5 . There is thus a blow-up $\widetilde{X} \rightarrow X$, and a surjective morphism of degree 5 :

$$
\psi: \widetilde{X} \rightarrow Y
$$

As $X$ has a small number of ordinary quadratic singularities, one can check using monodromy and vanishing cycles arguments that for any smooth birational model $Z$ of $X, H_{B}^{*}(Z, \mathbb{Z})$ has no torsion (cf. [6]). Hence $H_{B}^{*}(\widetilde{X}, \mathbb{Z})$ has no torsion and the existence of $\psi$, with $\psi_{*} \circ \psi^{*}=5 I d_{H^{*}(Y, \mathbb{Z})}$ show that the possible torsion of $H_{B}^{*}(Y, \mathbb{Z})$ has order 5 . Because the torsion of $H_{B}^{*}(S, \mathbb{Z})$ has order 2, we conclude that we have the surjectivity of the Künneth map:

$$
H_{B}^{*}(Y, \mathbb{Z}) \otimes H_{B}^{*}(S, \mathbb{Z}) \rightarrow H_{B}^{*}(W, \mathbb{Z})
$$


In degree 5 , we thus get that $H_{B}^{5}(W, \mathbb{Z})$ is a quotient of

$$
H_{B}^{5}(Y, \mathbb{Z}) \oplus H_{B}^{3}(Y, \mathbb{Z}) \otimes H_{B}^{2}(S, \mathbb{Z}) \oplus H_{B}^{2}(Y, \mathbb{Z}) \otimes H_{B}^{3}(S, \mathbb{Z}) .
$$

The cohomology group $H_{B}^{5}(Y, \mathbb{Z})=H_{1, B}(Y, \mathbb{Z})$ has coniveau 2 by Lefschetz theorem on hyperplane sections: Any smooth curve $j: D \hookrightarrow Y$ complete intersection of ample surfaces in $Y$ has the property that $j_{*}: H_{1, B}(D, \mathbb{Z}) \rightarrow H_{1, B}(Y, \mathbb{Z})$ is surjective. $H_{B}^{2}(S, \mathbb{Z})$ has coniveau 1 , and $H_{B}^{3}(Y, \mathbb{Q}$ ) has been assumed to have coniveau 1 (as predicted by the Grothendieck generalized Hodge conjecture). This implies that $H_{B}^{3}(Y, \mathbb{Z})$ has coniveau 1 because the sheaf $\mathcal{H}_{Y}^{3}(\mathbb{Z})$ has no torsion by Theorem 1.4. Finally $H_{B}^{2}(Y, \mathbb{Z})$ has coniveau 1 by Lefschetz theorem on $(1,1)$ classes and $H_{B}^{2}\left(Y, \mathcal{O}_{Y}\right)=0$ (cf. [8, Prop. 5.7]), and $H_{B}^{3}(S, \mathbb{Z})$ has coniveau 1 again by the Lefschetz hyperplane section theorem.

Thus the two summands $H_{B}^{3}(Y, \mathbb{Z}) \otimes H_{B}^{2}(S, \mathbb{Z})$ and $H_{B}^{2}(Y, \mathbb{Z}) \otimes H_{B}^{3}(S, \mathbb{Z})$ also have coniveau 2 , and thus $H_{B}^{5}(W, \mathbb{Z})=N^{2} H_{B}^{5}(W, \mathbb{Z})$.

The fact that $\mathrm{CH}_{0}(W)$ is supported on a threefold then implies by Corollary 0.3 that $\mathcal{T}^{3}(W) \cong H_{n r}^{4}(W, \mathbb{Q} / \mathbb{Z})$.

It thus only remains to show that $\mathcal{T}^{3}(W) \neq 0$.

We consider the variety $\widetilde{X} \times S$. $W$ is birationally equivalent to a quotient of $\widetilde{X} \times S$ by $G=\mathbb{Z} / 5 \mathbb{Z}$. We claim that it suffices now to exhibit an element $z$ of $\mathcal{T}^{3}(\widetilde{X} \times S)$ which is non zero, of order 2 and $G$-invariant. Indeed, let $\psi: \widetilde{X} \times S \rightarrow W$ be the morphism induced by $\phi: \widetilde{X} \rightarrow Y$. we conclude from birational invariance and from the invariance of $z$ under $G$ that $\psi^{*}\left(\psi_{*} z\right)=5 z$, which is nonzero since $z$ is of order 2 . Thus $\psi_{*} z \neq 0$ in $\mathcal{T}^{3}(W)$.

Let us exhibit a 2-torsion element $z \in \operatorname{Ker}_{c l}^{3} \subset C H^{3}(\tilde{X} \times S)$. The variety $\tilde{X}$ contains the curve $C_{0}$ which does not pass through the exceptional divisors over the singular points of $X$.

Lemma 4.5. There exists a G-invariant 1-cycle $C_{0}^{\prime}$ of $\widetilde{X}$ such that

$$
C_{0}^{\prime}=\sum_{g \in G} g\left(C_{0}\right)+D_{0}
$$

with $D_{0}$ supported in the fibers of $p: \widetilde{X} \rightarrow \mathbb{P}_{x y}^{1}$, and such that the class $\left[C_{0}^{\prime}\right] \in H^{4}(\widetilde{X}, \mathbb{Z})$ is divisible by 2 .

Proof. As we discussed above, $H^{*}(\widetilde{X}, \mathbb{Z})$ has no torsion, and furthermore $H^{2}\left(X, \mathcal{O}_{X}\right)=0$. It follows that there is a perfect pairing between $H^{4}(\widetilde{X}, \mathbb{Z})$ and $H^{2}(\widetilde{X}, \mathbb{Z})$, the latter being isomorphic to Pic $\widetilde{X}$. It thus suffices to show that there is a curve $C_{0}^{\prime}=\sum_{g \in G} g\left(C_{0}\right)+D_{0}$ with $D_{0}$ supported in the fibers of $p: \widetilde{X} \rightarrow \mathbb{P}_{x y}^{1}$ such that the degree of $C_{0}^{\prime}$ with respect to any divisor of $\widetilde{X}$ is even. But by [6] and the independence of the double points of $X, \operatorname{Pic} \widetilde{X}$ is generated by $\operatorname{Pic} X=\operatorname{Pic}\left(\mathbb{P}^{1} \times \mathbb{P}^{3}\right)$ and by the exceptional divisors of the desingularization $\widetilde{X} \rightarrow X$.

By construction, $C_{0}$ does not meet the exceptional divisors and $p^{*} c_{1}\left(\mathcal{O}_{\mathbb{P}_{x y}^{1}}(1)\right)$ has even degree on $C_{0}$. If it has odd degree on the remaining generator $p_{2}^{*} c_{1}\left(\mathcal{O}_{\mathbb{P}^{3}}(1)\right)$, we just add to $C_{0}$ an odd degree curve $\Delta$ in one fiber not passing through the singular points of $X$ (recall that $X$ is a one parameter family of $K 3$ surfaces, so the fibers containing an odd degree curve are topologically dense in the base $\left.\mathbb{P}^{1}\right)$. At this point, the constructed curve $C_{0} \cup \Delta$ is not $G$-invariant, but if we let $D_{0}=\cup_{g \in G} g(\Delta)$, we find that $C_{0}^{\prime}=\sum_{g \in G} g\left(C_{0}\right)+D_{0}$ satisfies the desired conclusion.

Let now $\eta$ be the generator of the 2-torsion of $H^{2}(S, \mathbb{Z})$. As Pic $S=N S(S)=H_{B}^{2}(S, \mathbb{Z}), \eta$ can be seen as a 2-torsion element of $C H^{1}(S)$. Let

$$
p r_{1}: \tilde{X} \times S \rightarrow \widetilde{X}, p r_{2}: \widetilde{X} \times S \rightarrow S
$$


be the two projections.

Lemma 4.6. The element $z:=p r_{1}^{*} C_{0}^{\prime} \cdot p r_{2}^{*} \eta \in C H^{3}(\widetilde{X} \times S)$ is of 2-torsion and has vanishing Deligne cycle class.

Proof. The cycle $z$ is of 2 -torsion because $\eta$ is. It is annihilated by the Deligne cycle class of $\widetilde{X} \times S$ for the following reason: The Betti cycle class of $C_{0}^{\prime}$ is divisible by 2 by Lemma 4.5 , which implies that the Deligne cycle class of $C_{0}^{\prime}$ is divisible by 2 because the kernel of the map

$$
H_{\mathcal{D}}^{4}(\widetilde{X}, \mathbb{Z}(2)) \rightarrow H_{B}^{4}(\widetilde{X}, \mathbb{Z})
$$

is a divisible group. As $\eta$ is of 2 -torsion, so is $c l_{\mathcal{D}}(\eta)$, and it follows that

$$
c l_{\mathcal{D}}(z)=\operatorname{pr}_{1}^{*}\left(c_{\mathcal{D}}\left(C_{0}^{\prime}\right)\right) \cdot \operatorname{pr}_{2}^{*}\left(c l_{\mathcal{D}}(\eta)\right)=0 \text { in } H_{\mathcal{D}}^{6}(\widetilde{X} \times S, \mathbb{Z}(2)) .
$$

In order to conclude, it remains to prove the following:

Lemma 4.7. The cycle $z \in C H^{3}(\widetilde{X} \times S)$ is nontrivial in $\operatorname{Griff}^{3}(\widetilde{X} \times S)$.

Proof. First of all, it obviously suffices to show that the cycle $p r_{1}^{*} C_{0}^{\prime} \cdot p r_{2}^{*} \eta \in C H^{3}(X \times S)$ (that we will also denote $z$ ) is nontrivial in $\operatorname{Griff}^{3}(X \times S)$. As in [20], we note that it suffices to prove that $z$ is not divisible by 2 , as the group of cycles algebraically equivalent to 0 is divisible. As $X$ is very general, the pair $\left(X, C_{0}^{\prime}\right)$ specializes to the pair $\left(X_{0}, C_{0}^{\prime}\right)$, and it suffices by $[20$, Lemma 3] to show that the cycle

$$
z_{0}:=p r_{1}^{*}\left(C_{0}^{\prime}\right) \cdot p r_{2}^{*} \eta \in C H_{2}\left(X_{0} \times S\right)
$$

is not divisible by 2 . Note that the product here is well defined by $[10,2.3]$, as $p r_{2}^{*} \eta$ belongs to $\operatorname{Pic}\left(X_{0} \times S\right)$.

We now observe the following: the variety $X_{0} \subset \mathbb{P}_{x y}^{1} \times \mathbb{P}^{3}$ is the image via a morphism

$$
\left(\phi^{\prime}, I d\right): \mathbb{P}^{1} \times \mathbb{P}^{3} \rightarrow \mathbb{P}_{x y}^{1} \times \mathbb{P}^{3}
$$

of an hypersurface $X_{0}^{\prime} \subset \mathbb{P}^{1} \times \mathbb{P}^{3}$ of bidegree $(3,2)$, and furthermore the curve $C_{0}^{\prime}$ comes from a curve $C_{0}^{\prime \prime} \subset X_{0}^{\prime}$. To see this, it suffices to recall that

$$
X_{0}=(\phi, I d)(\Gamma)
$$

where $\Gamma \subset \mathbb{P}_{u v}^{1} \times \mathbb{P}^{3}$ is an hypersurface of bidegree $(3,1)$, and $\phi: \mathbb{P}_{u v}^{1} \rightarrow \mathbb{P}_{x y}^{1}$ is a Galois cover of group $\mathbb{Z} / 4 \mathbb{Z}$. We thus factor $\phi$ as $\phi^{\prime} \circ \phi^{\prime \prime}$, where both $\phi^{\prime}: \mathbb{P}^{1} \rightarrow \mathbb{P}_{x y}^{1}$ and $\phi^{\prime \prime}: \mathbb{P}_{u v}^{1} \rightarrow \mathbb{P}^{1}$ are of degree 2. Denoting $X_{0}^{\prime}:=\left(\phi^{\prime \prime}, I d\right)(\Gamma)$, it just suffices to remember that the curve $C_{0}$ was precisely defined as the image via $\left(\phi^{\prime}, I d\right)$ of a curve contained in $\Sigma_{0}$, the later being naturally contained in $X_{0}^{\prime}$.

The cycle $z_{0}^{\prime}:=p r_{1}^{*}\left(C_{0}^{\prime \prime}\right) \cdot p r_{2}^{*} \eta \in C H_{2}\left(X_{0}^{\prime} \times S\right)$ is not divisible by 2 in $C H_{2}\left(X_{0}^{\prime} \times S\right)$. Indeed, $X_{0}^{\prime}$ admits a morphism $p^{\prime}: X_{0}^{\prime} \rightarrow \mathbb{P}^{1}$, and the degree of $p_{\mid C_{0}^{\prime \prime}}^{\prime}: C_{0}^{\prime \prime} \rightarrow \mathbb{P}^{1}$ is 5 . It follows that

$$
p r_{2 *}\left(z_{0}^{\prime} \cdot p^{\prime *} c_{1}\left(\mathcal{O}_{\mathbb{P}^{1}}(1)\right)=5 \eta=\eta \text { in } C H_{1}(S),\right.
$$

and as $\eta$ is not divisible by 2 in $C H_{1}(S), z_{0}^{\prime}$ is not divisible by 2 in $C H_{2}\left(X_{0}^{\prime} \times S\right)$. The morphism $\left(\phi^{\prime}, I d\right): X_{0}^{\prime} \rightarrow X_{0}$ is two-to-one on the image $\Sigma_{1}^{\prime \prime} \subset X_{0}^{\prime}$ of the surface $\Sigma_{1}^{\prime} \subset X_{0}$ introduced in (4.26), and otherwise one-to-one. It is easy to see that any curve $Z \subset \Sigma_{1}^{\prime \prime}$ has degree divisible by 2 over $\mathbb{P}^{1}$. It follows that for any 2-cycle $Z$ of $X_{0}^{\prime} \times S$ supported on $S \times \Sigma_{1}$, we have

$$
\operatorname{pr}_{2 *}\left(Z \cdot p^{\prime *} c_{1}\left(\mathcal{O}_{\mathbb{P}^{1}}(1)\right)=0 \text { in } C H_{1}(S) \otimes \mathbb{Z} / 2 \mathbb{Z}\right. \text {. }
$$

Now, if $z_{0}$ was divisible by 2 in $\mathrm{CH}_{2}\left(\mathrm{X}_{0} \times S\right)$, $z_{0}^{\prime}$ would be divisible by 2 in $\mathrm{CH}_{2}\left(\mathrm{X}_{0}^{\prime} \times S\right) / \mathrm{CH}_{2}(S \times$ $\left.\Sigma_{1}^{\prime \prime}\right)$ and this contradicts (4.27) and (4.28). 
The proof of Proposition 4.4 is finished.

\section{References}

[1] M. Artin, D. Mumford. Some elementary examples of unirational varieties which are not rational , Proc. London Math. Soc. (3) 25 (1972), 7595.

[2] L. Barbieri-Viale, On the Deligne-Beilinson cohomology sheaves, prépublication, arXiv: alg-geom/9412006v1.

[3] S. Bloch, A. Kas, D. Lieberman. Zero cycles on surfaces with $p_{g}=0$. Compositio Math. 33 (1976), no. 2, 135-145.

[4] S. Bloch, A. Ogus. Gersten's conjecture and the homology of schemes, Ann. Sci. Éc. Norm. Supér., IV. Sér. 7, 181-201 (1974).

[5] S. Bloch, V. Srinivas. Remarks on correspondences and algebraic cycles, Amer. J. of Math. 105 (1983) 1235-1253.

[6] H. Clemens. Double solids. Adv. in Math. 47 (1983), no. 2, 107-230.

[7] J.-L. Colliot-Thélène, M. Ojanguren. Variétés unirationnelles non rationnelles: au-delà de l'exemple d'Artin et Mumford, Invent. math. 97 (1989), no. 1, 141-158.

[8] J.-L. Colliot-Thélène, C. Voisin. Cohomologie non ramifiée et conjecture de Hodge entière,

[9] P. Deligne. Théorie de Hodge. II, Inst. Hautes Études Sci. Publ. Math. (1971), no. 40, 5-57.

[10] W. Fulton. Intersection Theory, Ergebnisse der Math. und ihrer Grenzgebiete 3 Folge, Band 2, Springer (1984).

[11] H. Gillet, C. Soulé. C. Filtrations on higher algebraic $K$-theory. Algebraic $K$-theory (Seattle, WA, 1997), 89-148, Proc. Sympos. Pure Math., 67, Amer. Math. Soc., Providence, RI, (1999).

[12] P. Griffiths. On the periods of certain rational integrals I,II, Ann. of Math. 90 (1969), 460-541.

[13] A. Grothendieck. Hodge's general conjecture is false for trivial reasons, Topology 8 (1969) 299-303.

[14] Yu. Manin. Correspondences, motifs and monoidal transformations. (Russian) Mat. Sb. (N.S.) 77 (119), 475-507 (1968).

[15] J. P. Murre. Applications of algebraic K-theory to the theory of algebraic cycles, in Proc. Conf. Algebraic Geometry, Sitjes 1983, LNM 1124 (1985), 216-261, Springer-Verlag.

[16] M. Nori. Algebraic cycles and Hodge-theoretic connectivity. Invent. Math. 111 (1993), no. 2, 349-373.

[17] E. Peyre. Unramified cohomology and rationality problems, Math Ann. 296 (1993), 247-268.

[18] C. Schoen. Some examples of torsion in the Griffiths group. Math. Ann. 293 (1992), no. 4, 651-679. 
[19] C. Schoen. Specialization of the torsion subgroup of the Chow group, Math. Z. 252 (2006), no. $1,11-17$.

[20] C. Soulé, C. Voisin. Torsion cohomology classes and algebraic cycles on complex projective manifolds. Adv. Math. 198 (2005), no. 1, 107-127.

[21] J. Starr, A pencil of Enriques surfaces of index one with no section, Algebra and Number Theory 3 (2009) no. 6, 637-652.

[22] C. Voisin. Sur les zéro-cycles de certaines hypersurfaces munies d'un automorphisme. Ann. Scuola Norm. Sup. Pisa Cl. Sci. (4) 19 (1992), no. 4, 473-492.

[23] C. Voisin. Hodge Theory and Complex Algebraic Geometry I, II, Cambridge studies in advanced Mathematics 76, 77, Cambridge University Press 2002, 2003. 